Schwerpunkt

Kardiologe 2021 $15: 160-167$

https://doi.org/10.1007/s12181-021-00467-8

Angenommen: 3. Februar 2021

Online publiziert: 10. März 2021

(C) Deutsche Gesellschaft für Kardiologie -

Herz- und Kreislaufforschung e.V. Published by

Springer Medizin Verlag $\mathrm{GmbH}$, ein Teil von

Springer Nature - all rights reserved 2021

\author{
Dominic Millenaar ${ }^{1}$ - Christian Ukena' ${ }^{1}$ Andreas Keller ${ }^{2} \cdot$ Felix Mahfoud' $^{1}$. \\ Michael Böhm ${ }^{1}$ \\ ' Klinik für Innere Medizin III, Kardiologie, Angiologie und Internistische Intensivmedizin, \\ Universitätsklinikum des Saarlandes und Medizinische Fakultät der Universität des Saarlandes, \\ Homburg/Saar, Deutschland \\ ${ }^{2}$ Zentrum für Bioinformatik Saar, Abteilung für klinische Bioinformatik, Universität des Saarlandes, \\ Saarbrücken, Deutschland
}

\title{
Kardiovaskuläre Forschung in Deutschland 2020 - eine szientometrische Analyse
}

(WoS) im Jahr 1972 bis einschließlich 2020. Hierfür wurden geeignete Suchbegriffe erstellt [2], um alle relevanten Publikationen einzuschließen. Weltweit konnten hierdurch insgesamt 1.059.530 Publikationen im Bereich der kardiovaskulären Medizin gefunden werden, davon stammten 55.062 Veröffentlichungen aus Forschungsinstituten in Deutschland. Die Publikationen wurden mit den zugehörigen Metadaten von WoS extrahiert und mittels des von uns entwickelten Webtools SciPE (Science Performance Evaluation) analysiert [5]. Hierbei erfolgte eine Geokodierung der Datensätze mit entsprechender Zuordnung der jeweiligen Publikationen $\mathrm{zu}$ den Forschungsinstituten. Anhand dieser Daten konnte eine Heatmap aller beteiligten Institute erstellt werden (•Abb. 1), welche die Forschungsleistung des gesamten Bundesgebietes farbkodiert visualisiert. Weiterhin erfolgte eine Zuordnung zu den einzelnen Bundesländern, wie beispielsweise in -Abb. 2 dargestellt ist. Zur Beurteilung der Forschungsqualität wurde der durchschnittliche Hirsch(H)Index ermittelt. Der H-Index beschreibt die Anzahl der Publikationen $\mathrm{h}$ eines Autors, die h-fach zitiert wurden [3]. In einer modifizierten Version kann der H-Index auch auf Institute, Bundesländer und sogar Länder ausgeweitet werden. Weiterhin erfolgte eine Analyse nach verschiedenen Themenbereichen der Kardiologie, wie beispielsweise der koronaren Herzkrankheit oder Herzin- suffizienz. Hierfür wurde der Suchbegriff entsprechend angepasst, um die geeigneten Publikationen eines jeden Themas einzeln extrahieren und analysieren zu können. Eine Geschlechteranalyse konnte mithilfe der Zuordnung jedes Erstautors zu entweder „weiblich“ oder „männlich“ erlangt werden. Die Zuordnung erfolgte automatisiert anhand des Vornamens. In den Fällen, in denen der Vorname nicht vollständig angegeben oder sowohl weiblich als auch männlich einzustufen war, blieb das Geschlecht unbekannt. Diese Publikationen wurden bei der genauen Geschlechteranalyse nicht berücksichtigt.

\section{Forschung in unterschiedlichen Themenbereichen}

Zur weiteren Analyse der kardiovaskulären Forschung erfolgte eine getrennte Untersuchung nach häufigen Themenbereichen in der Kardiologie (• Tab. 1). Die Forschungsaktivität im Bereich der KHK war mit insgesamt 6153 Publikationen am größten, während im Bereich der pAVK 2261 Originalarbeiten publiziert wurden. Die - Abb. 2 und 3 visualisieren die Verteilung der Gesamtanzahl aller Publikationen dieser einzelnen Themenbereiche in den verschiedenen Bundesländern. Hierbei wird deutlich, dass Bundesländer mit einer großen Einwohnerzahl und einer hohen Anzahl an medizinischen Fakultäten entsprechend die meisten Publikationen veröffentlichten. Beispielhaft können hier 


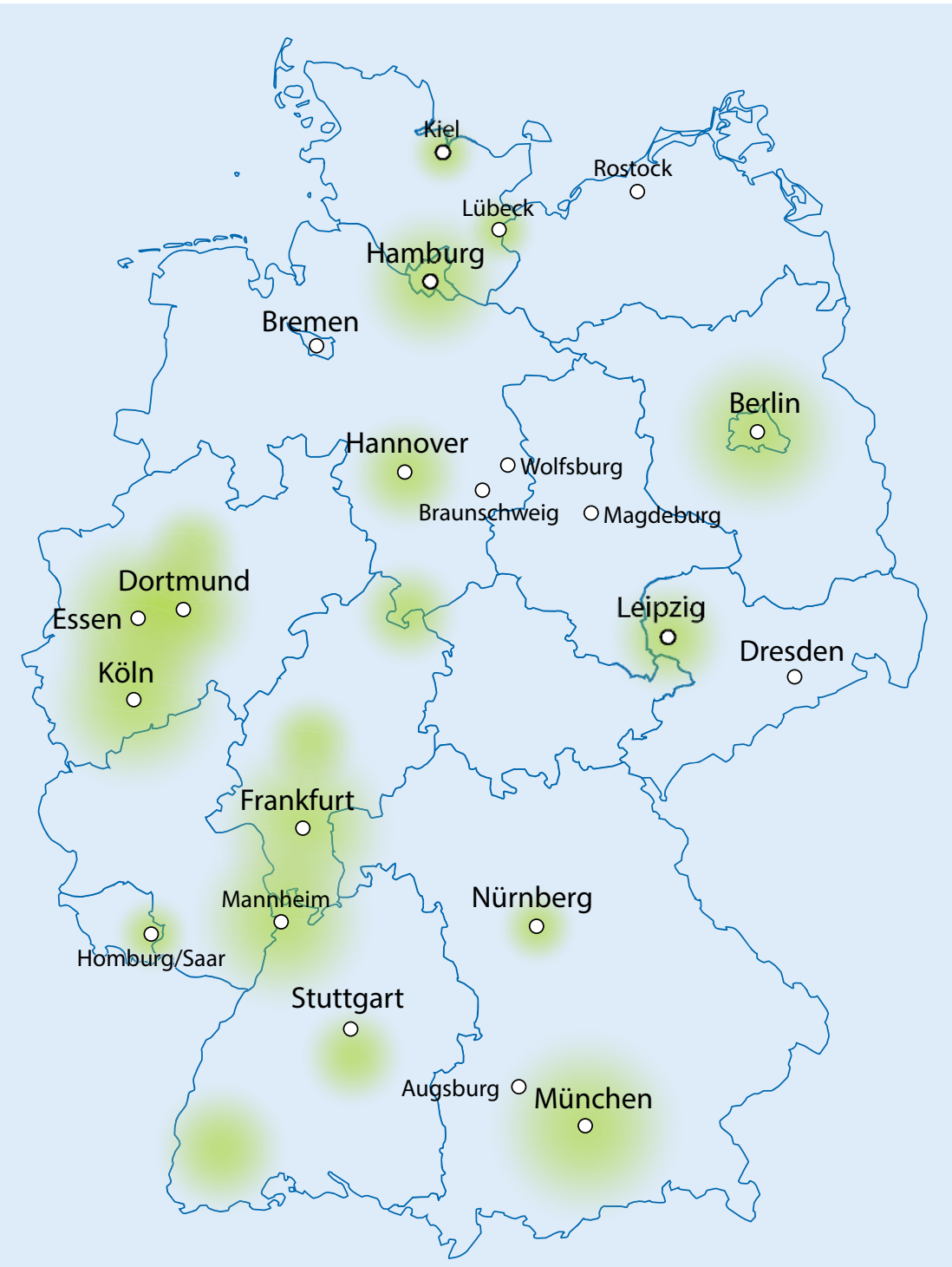

Abb. $1 \Delta$ Heatmap aller Forschungsinstitute in Deutschland. In grün ist die Anzahl aller Publikationen der jeweiligen Institute abgebildet, die Farbintensität ist proportional zur absoluten Anzahl an Veröffentlichungen

im Themengebiet der KHK das Bundesland Bayern mit 1394 Publikationen und Nordrhein-Westfalen mit 1082 Publikationen genannt werden. Zur besseren Übersicht sollten die Publikationen relativ $\mathrm{zu}$ anderen Parametern betrachtet werden, wie in $\square$ Tab. 1 dargestellt. Hier wurden die Einwohnerzahl, das Bruttoinlandsprodukt (BIP) sowie die Anzahl der medizinischen Fakultäten genannt. Durch die Berücksichtigung dieser Ressourcen - sowohl finanziell als auch infrastrukturell - lassen sich die Publikationsleistungen einheitlicher vergleichen. Insbesondere Bundesländer mit einer ge-

here Anzahl an Publikationen auch die Wahrscheinlichkeit für eine höhere Zahl an Zitationen der Arbeiten steigt, kann hierdurch die wissenschaftliche Relevanz der Arbeiten besser eingeschätzt werden. Den höchsten durchschnittlichen H-Index weisen die Themen Arrhythmien und
KHK auf (je 10,1), während die pAVK nur einen durchschnittlichen Wert von 5,2 erzielte.

\section{Geschlechteranalyse}

Wie bereits beschrieben [2], ist der Frauenanteil in der kardiovaskulären Forschung (noch) geringer als der männlicher Autoren. Innerhalb der verschiedenen Themengebiete der Kardiologie ist diese Verteilung jedoch sehr unterschiedlich, wie $\bullet$ Abb. 4 zeigt. Im Bereich der Arrhythmien (17\%) sowie der KHK $(18 \%)$ ist der prozentuale Frauenanteil am kleinsten, während im Bereich der pAVK $(24 \%)$ und Herzinsuffizienz (25\%) rund ein Viertel aller Erstautoren Frauen waren. Wie in 0 Tab. 2 dargestellt, ist der durchschnittliche H-Index aufgrund der geringeren Anzahl an Publikationen ebenfalls geringer bei $\mathrm{Au}$ torinnen, jedoch weniger ausgeprägt als im Vergleich der Publikationszahlen. Bei der Forschung zur pAVK war der geringste Unterschied zwischen Männern und Frauen zu verzeichnen (H-Index 4,1 vs. 6,8). Dies spiegelt sich auch in der höheren Erfolgsrate junger Kardiologinnen in Deutschland bei der Publikation in hochrangigen Fachzeitschriften wider [6].

Der Frauenanteil im Bereich der kardiovaskulären Forschung hat sich in den letzten 20 Jahren deutlich gesteigert. Wie in Abb. 5 gezeigt, liegt der Anteil der weiblichen Autorinnen in allen Publikationen zwischen 2001 und 2010 bei $15 \%$, während dieser Anteil zwischen 2011 und 2020 auf $26 \%$ gestiegen ist. Dieser Anstieg lässt sich in allen 6 separat analysierten Themenbereichen beobachten. Eine Analyse des $\mathrm{H}$-Indexes im zeitlichen Verlauf ist nicht sinnvoll, da der H-Index mit der Anzahl der Zitationen einer Arbeit steigt und somit ältere Publikationen regelhaft einen höheren $\mathrm{H}$-Index aufweisen.

\section{Kooperationen}

Zur Analyse der internationalen Kooperationen wurden innerhalb der Publikationen mit deutschem Erstautor die Kooperationen zu den Ländern der Koautoren ausgewertet und grafisch in einem 
Kardiologe 2021 · 15:160-167 https://doi.org/10.1007/s12181-021-00467-8

๑) Deutsche Gesellschaft für Kardiologie - Herz- und Kreislaufforschung e.V. Published by Springer Medizin Verlag GmbH, ein Teil von Springer Nature all rights reserved 2021

\section{Millenaar · C. Ukena · A. Keller · F. Mahfoud · M. Böhm}

\section{Kardiovaskuläre Forschung in Deutschland 2020 - eine szientometrische Analyse}

\section{Zusammenfassung}

In der vorliegenden szientometrischen Analyse wurde die Forschungsleistung im Bereich der kardiovaskulären Medizin untersucht unter Berücksichtigung der zunehmenden Subspezialisierung. Hierbei zeigte sich, dass die Forschungsaktivität im Bereich der koronaren Herzkrankheit (KHK) mit 6153 Publikationen am größten war, während sich beispielsweise im Bereich der peripheren arteriellen Verschlusskrankheit ( $p A V K)$ eine geringere Aktivität mit 2261 Originalarbeiten zeigte. Es erfolgte eine weitere Untersuchung der Forschungsleistung getrennt nach allen Bundesländern (sowohl bezogen auf die Einwohnerzahl als auch das Bruttoinlandsprodukt [BIP] sowie die
Anzahl medizinischer Fakultäten), bei der sich thematische Schwerpunkte verzeichnen ließen. Die Qualität der kardiovaskulären Forschung wurde anhand des Hirsch(H)-Indexes bestimmt. Hierbei wiesen die Themenbereiche KHK und Arrhythmien mit einem $\mathrm{H}$-Index von 10,1 den höchsten Wert auf. Darüber hinaus erfolgte eine geschlechterspezifische Analyse der kardiologischen Subspezialisierungen, bei der ein deutlicher Unterschied zwischen den verschiedenen Themenbereichen festgestellt werden konnte. Der Frauenanteil war mit 17\% im Bereich der Arrhythmien am niedrigsten, während der Bereich der Herzinsuffizienz mit 25\% den größten Anteil an Frauen aufwies. In allen analysierten Bereichen kam es innerhalb der letzten 20 Jahre zu einem deutlichen Zuwachs weiblicher Autorinnen. Der durchschnittliche $\mathrm{H}$-Index war bei Frauen ebenfalls geringer, jedoch war der Unterschied hier weniger ausgeprägt als bei der absoluten Zahl an Publikationen. Weiterhin konnten häufige internationale Kooperationen festgestellt werden, wobei die USA in allen Themenbereichen den häufigsten Kooperationspartner Deutschlands darstellte, gefolgt von zahlreichen europäischen Nachbarländern.

\section{Schlüsselwörter}

Kardiologie · Zitationsanalyse $\cdot$ Geschlecht . Subspezialisierung $\cdot$ Kooperationen

\section{Cardiovascular research in Germany 2020_A scientometric analysis}

\section{Abstract}

In the present scientometric analysis, the research performance in the field of cardiovascular medicine was examined, taking the increasing subspecialization into account. The analysis showed that research activity was highest in the field of coronary artery disease (CAD) with 6153 publications, whereas, for example, the field of peripheral artery occlusive disease (PAOD) showed lower activity with 2261 original articles. A further examination of the research performance was carried out separately for all federal states (both in terms of the number of inhabitants and the gross domestic product, GDP, as well as the number of medical faculties) in which thematic focusses could be noted. The quality of cardiovascular research was determined using the Hirsch $(\mathrm{H})$ index. Here, the fields of CAD and arrhythmia showed the highest scores with an $\mathrm{H}$ index of 10.1. In addition, a gender-specific analysis of cardiovascular subspecialties was performed, which revealed a clear difference between the various subject areas. The percentage of women was lowest in research in the field of arrhythmia (17\%) compared to the field of heart failure with the highest proportion of women (25\%). In all analyzed subspecialties, there has been a significant increase in female authors within the last 20 years. The average $\mathrm{H}$ index was also lower for women but the difference was less pronounced here than in the absolute number of publications. Furthermore, international collaborations were commonly observed, with the USA being Germany's most frequent cooperation partner in all subject areas, followed by numerous neighboring European countries.

Keywords

Cardiology · Citation analysis - Sex .

Subspecialization $\cdot$ Cooperations
Akkorddiagramm („chord diagram“) gezeigt (•Abb. 6). Die Kreisabschnitte eines jeden Landes sind proportional zur Anzahl der Kooperationen. Ebenso lassen sich anhand der Breite der Verbindungslinien zwischen den Ländern („chords“) Rückschlüsse auf das Ausmaß der Zusammenarbeit ziehen. Im Bereich der gesamten kardiovaskulären Forschung sind die Vereinigten Staaten von Amerika (USA) stets der häufigste Kooperationspartner für deutsche Forschungsprojekte. Im Bereich der Arrhythmien, der arteriellen Hypertonie sowie der Herzinsuffizienz stellte Großbritannien das dritthäufigste ko- operierende Land dar, im Bereich der KHK sowie pAVK waren dies die Niederlande, bei den Klappenvitien Italien. Weitere häufige Kollaborationen bestanden mit anderen europäischen Ländern, während asiatische Länder wie China oder Japan eine eher untergeordnete Rolle spielten.

\section{Limitationen}

Bei der vorliegenden szientometrischen Analyse müssen einige Limitationen berücksichtigt werden. Die gesamte Analyse basiert auf den Informationen, die zu Beginn von WoS extrahiert werden. Trotz
Gegenproben in anderen medizinischen Datenbanken wie PubMed stützt sich die Integrität der Daten auf die Inhalte von WoS. Ebenso sind diese abhängig vom gewählten Suchterminus. Bei der Zuordnung zu den einzelnen Regionen wurde für jede Publikation nur der Korrespondenzautor berücksichtigt, was zu einer Unterrepräsentation der Koautoren führen kann. Dies gilt ebenso für die Geschlechterzuteilung, die anhand der Vornamen desselben Autors erfolgte. Trotz Berücksichtigung länderspezifischer $\mathrm{Zu}$ ordnungen, lässt sich hier ein mögliches Bias nicht ausschließen. Wenn der Vorname des Korrespondenzautors nicht be- 


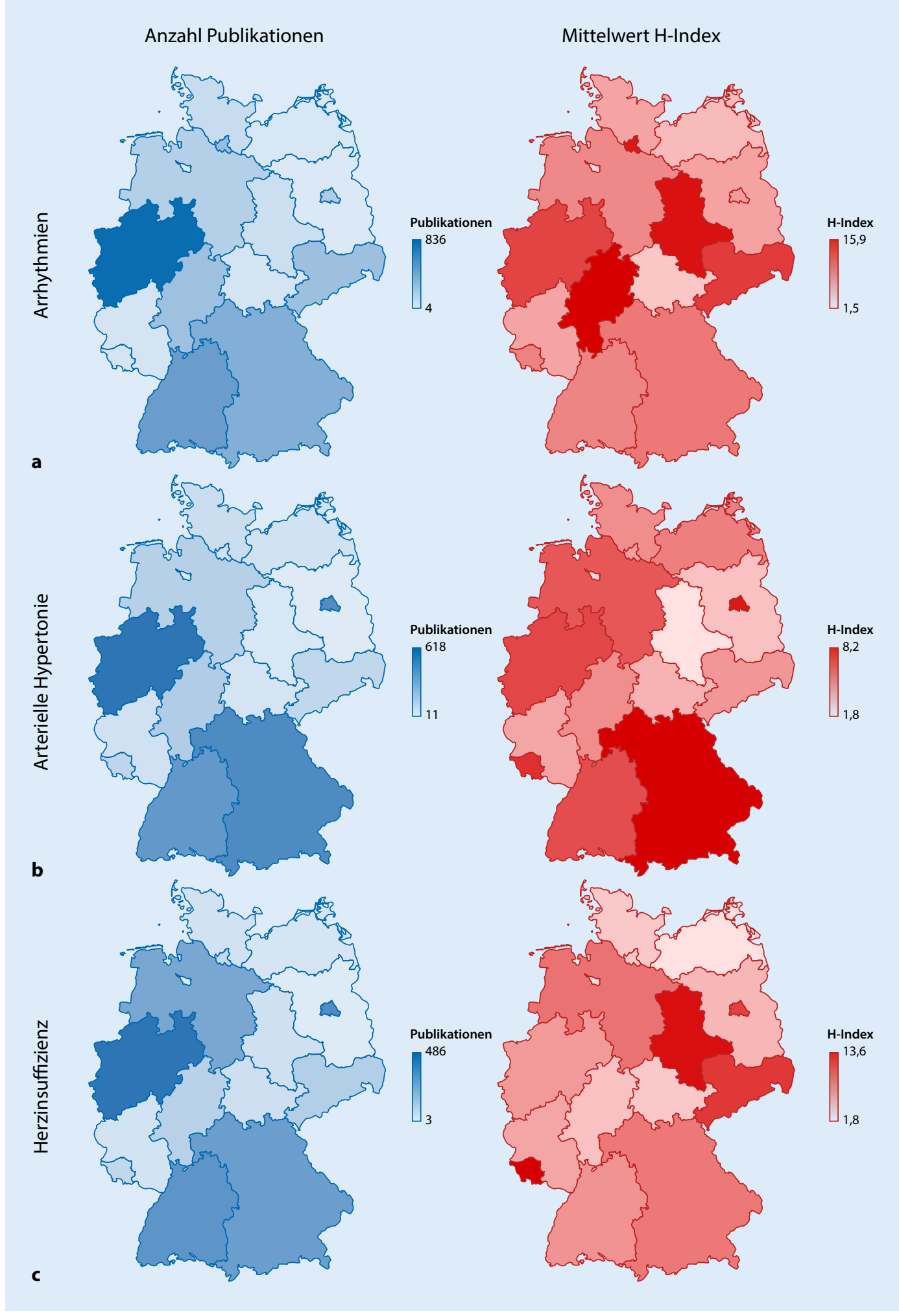

Abb. $2 \triangleleft$ Verteilung der kardiovaskulären Forschung in allen Bundesländern in Bezug auf die Anzahl der Publikationen (blau), sowie den durchschnittlichen Hirsch(H)Index (rot) für die Themengebiete Arrhythmien (a), arterielle Hypertonie (b) und Herzinsuffizienz (c) 


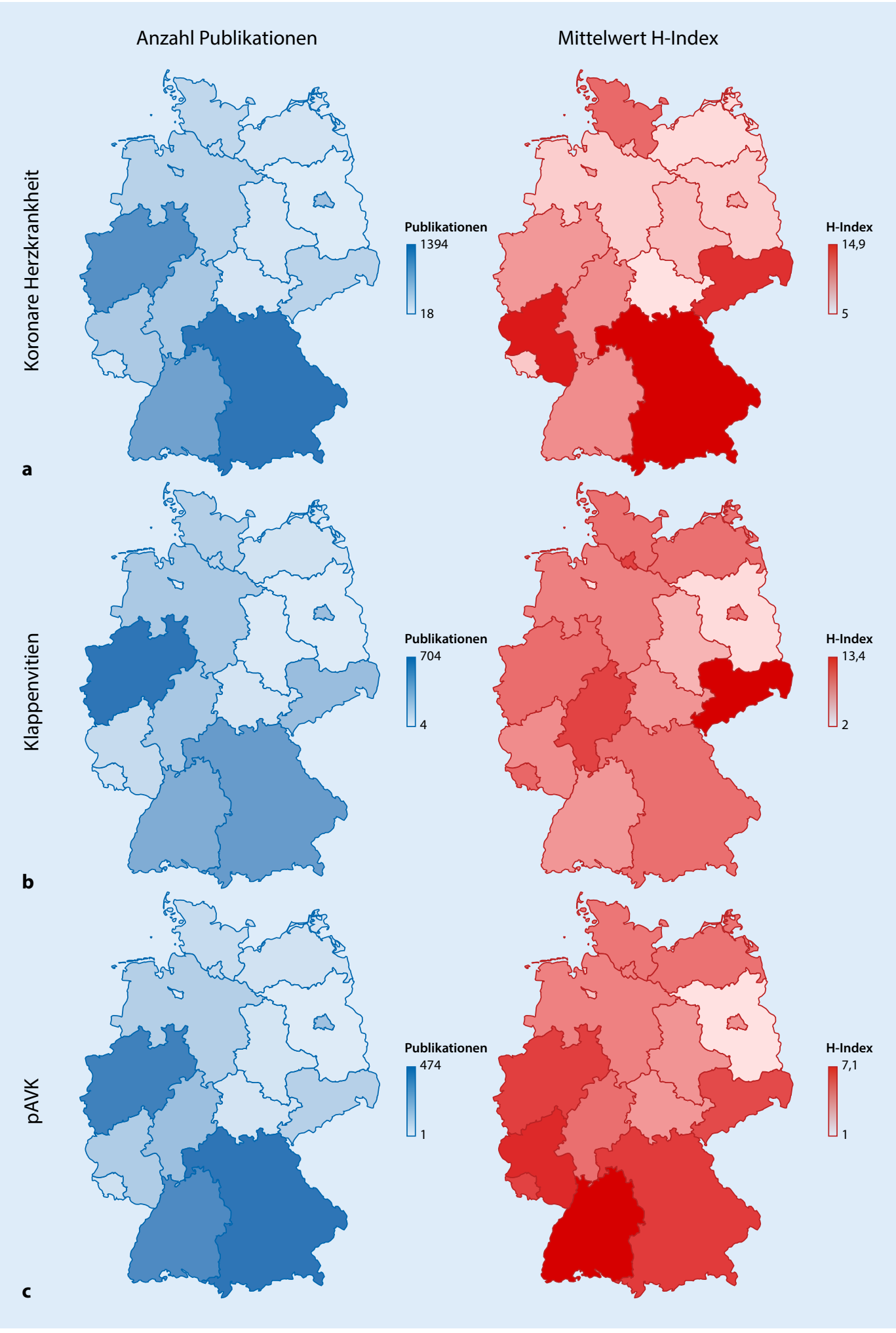

Abb. $3 \triangleleft$ Verteilung der kardiovaskulären Forschung in allen Bundesländern in Bezug auf die Anzahl der Publikationen (blau) sowie den durchschnittlichen $\mathrm{H}$-Index (rot) für die Themengebiete koronare Herzkrankheit (a), Klappenvitien (b) und periphere arterielle Verschlusskrankheit (pAVK) (c) 


\begin{tabular}{|c|c|c|c|c|c|c|c|c|c|c|}
\hline & \multirow[t]{2}{*}{ Bundesland } & \multirow{2}{*}{$\begin{array}{l}\text { Einwohner- } \\
\text { zahl (in Tau- } \\
\text { send) [7] }\end{array}$} & \multicolumn{6}{|c|}{ Anzahl der Publikationen } & \multirow{2}{*}{$\begin{array}{l}\text { BIP (in } \\
\text { Mio. €) [7] }\end{array}$} & \multirow{2}{*}{$\begin{array}{l}\text { Anzahl } \\
\text { medizi- } \\
\text { nischer } \\
\text { Fakultäten }\end{array}$} \\
\hline & & & $\begin{array}{l}\text { Arrhyth- } \\
\text { mien }\end{array}$ & $\begin{array}{l}\text { Arterielle Hyper- } \\
\text { tonie }\end{array}$ & $\begin{array}{l}\text { Herzinsuffi- } \\
\text { zienz }\end{array}$ & KHK & $\begin{array}{l}\text { Klappen- } \\
\text { vitien }\end{array}$ & PAVK & & \\
\hline 1 & Baden-Württemberg & 11.100 & 551 & 434 & 355 & 881 & 376 & 389 & 524.325 & 5 \\
\hline 2 & Bayern & 13.125 & 432 & 506 & 317 & 1394 & 478 & 474 & 632.897 & 6 \\
\hline 3 & Berlin & 3670 & 227 & 493 & 393 & 503 & 268 & 171 & 153.291 & 1 \\
\hline 4 & Brandenburg & 2522 & 16 & 11 & 5 & 23 & 4 & 1 & 74.330 & 0 \\
\hline 5 & Bremen & 681 & 4 & 18 & 3 & 18 & 4 & 1 & 33.623 & 0 \\
\hline 6 & Hamburg & 1847 & 338 & 62 & 74 & 216 & 148 & 74 & 123.270 & 1 \\
\hline 7 & Hessen & 6288 & 302 & 170 & 108 & 438 & 213 & 179 & 294.477 & 3 \\
\hline 8 & $\begin{array}{l}\text { Mecklenburg-Vor- } \\
\text { pommern }\end{array}$ & 1608 & 32 & 57 & 28 & 94 & 53 & 37 & 46.567 & 2 \\
\hline 9 & Niedersachsen & 7994 & 202 & 150 & 281 & 315 & 207 & 114 & 307.036 & 2 \\
\hline 10 & Nordrhein-Westfalen & 17.947 & 836 & 618 & 486 & 1082 & 704 & 427 & 711.419 & 7 \\
\hline 11 & Rheinland-Pfalz & 4094 & 51 & 62 & 35 & 410 & 100 & 127 & 145.003 & 1 \\
\hline 12 & Saarland & 987 & 62 & 122 & 83 & 65 & 51 & 41 & 36.253 & 1 \\
\hline 13 & Sachsen & 4072 & 310 & 115 & 121 & 302 & 278 & 109 & 63.545 & 2 \\
\hline 14 & Sachsen-Anhalt & 2195 & 89 & 33 & 45 & 75 & 16 & 27 & 128.097 & 2 \\
\hline 15 & Schleswig-Holstein & 2904 & 107 & 77 & 40 & 253 & 171 & 62 & 97.762 & 2 \\
\hline 16 & Thüringen & 2133 & 47 & 45 & 51 & 84 & 43 & 28 & 63.866 & 1 \\
\hline - & Deutschland & 83.191 & 3606 & 2973 & 2425 & 6153 & 3114 & 2261 & 3.449 .050 & 36 \\
\hline
\end{tabular}

Tab. 2 Anzahl und Hirsch(H)-Index der Publikationen nach Geschlecht

\begin{tabular}{|c|c|c|c|c|c|c|}
\hline & \multicolumn{3}{|c|}{ Gesamtzahl der Publikationen } & \multicolumn{3}{|c|}{$\begin{array}{l}\text { Durchschnittlicher H-Index pro } \\
\text { Publikation }\end{array}$} \\
\hline & Weiblich & Männlich & $\begin{array}{l}\text { \% weib- } \\
\text { lich }\end{array}$ & Weiblich & Männlich & $\%$ weiblich \\
\hline Arrhythmien & 508 & 2415 & 17,4 & 6,27 & 12,62 & 33,2 \\
\hline Arterielle Hypertonie & 462 & 1458 & 24,1 & 3,21 & 10,73 & 23,0 \\
\hline Herzinsuffizienz & 480 & 1448 & 24,9 & 4,60 & 9,34 & 33,0 \\
\hline Koronare Herzkrankheit & 879 & 3886 & 18,4 & 7,23 & 13,09 & 35,6 \\
\hline Klappenvitien & 503 & 2033 & 19,8 & 5,67 & 10,08 & 36,0 \\
\hline $\begin{array}{l}\text { Periphere arterielle } \\
\text { Verschlusskrankheit }\end{array}$ & 382 & 1192 & 24,3 & 4,07 & 6,81 & 37,4 \\
\hline
\end{tabular}

kannt war (z. B. bei Initialen), war ebenso keine Zuordnung möglich. Durch diese automatisierte Zuteilung der Publikationen auf Regionen oder Geschlechter wird hingegen eine umfangreiche Analyse erst möglich gemacht.

\section{Schlussfolgerung}

Die Anzahl an kardiovaskulären Publikationen hat in Deutschland sowie weltweit in den letzten Jahren deutlich zugenommen. Es zeigen sich klare Unterschiede innerhalb verschiedener Themenbereiche der Kardiologie in Bezug auf Publikationszahl, aber auch Publikations- qualität. Ebenso lassen sich in den verschiedenen Bundesländern thematische Schwerpunkte verzeichnen. Zur genaueren Analyse sollten weitere Faktoren wie Einwohnerzahl, BIP sowie Anzahl der medizinischen Fakultäten berücksichtigt werden. Es besteht bis heute eine Unterrepräsentation von Autorinnen, jedoch in unterschiedlicher Ausprägung je nach Themengebiet. Diese Lücke hat sich in den letzten Jahren bereits verkleinert. In Bezug auf die Publikationsqualität ist diese weniger ausgeprägt. Innerhalb kardiovaskulärer Publikationen bestehen häufige internationale Kooperationen, wobei die USA den häufigsten Kooperations- partner darstellen, gefolgt von zahlreichen europäischen Nachbarländern.

\section{Fazit für die Praxis}

- Die kardiovaskuläre Forschung nimmt weiterhin stetig zu.

- Unterschiede zeigen sich zwischen den einzelnen Subspezialisierungen der Kardiologie sowohl in den unterschiedlichen Bundesländern als auch zwischen Männern und Frauen.

- Internationale Kooperationen lassen sich in allen Bereichen deutlich erkennen. 


\section{Schwerpunkt}

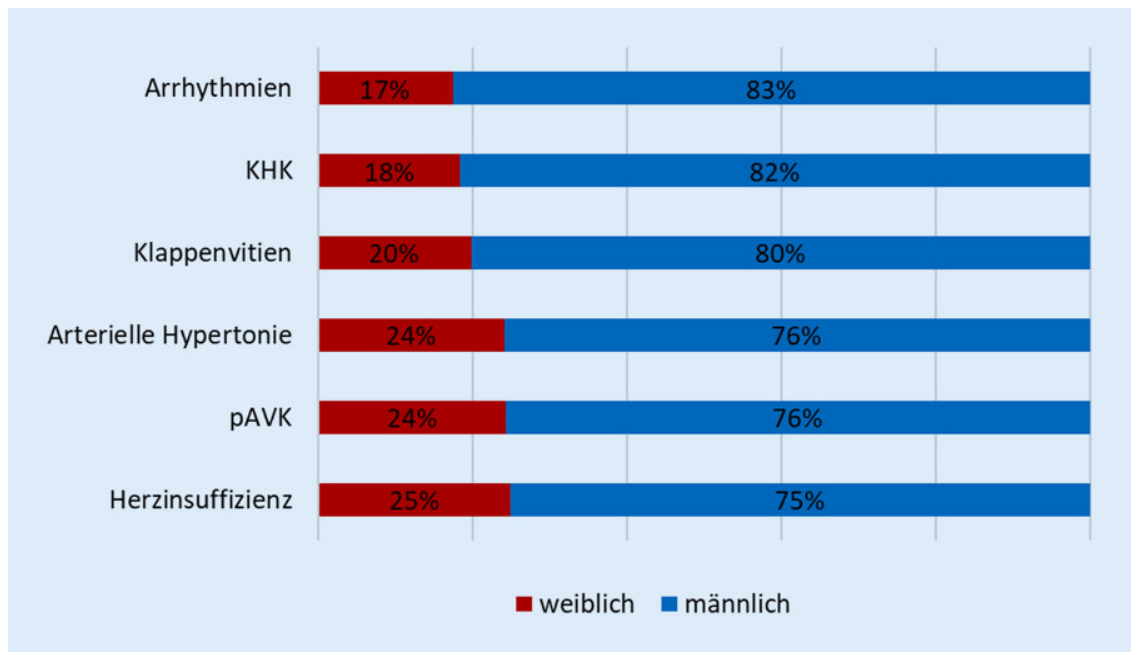

Abb. $4 \Delta$ Geschlechterverteilung der männlichen und weiblichen Autoren in der kardiologischen Forschung in Prozent bis 2020. pAVK periphere arterielle Verschlusskrankheit, KHKkoronare Herzkrankheit

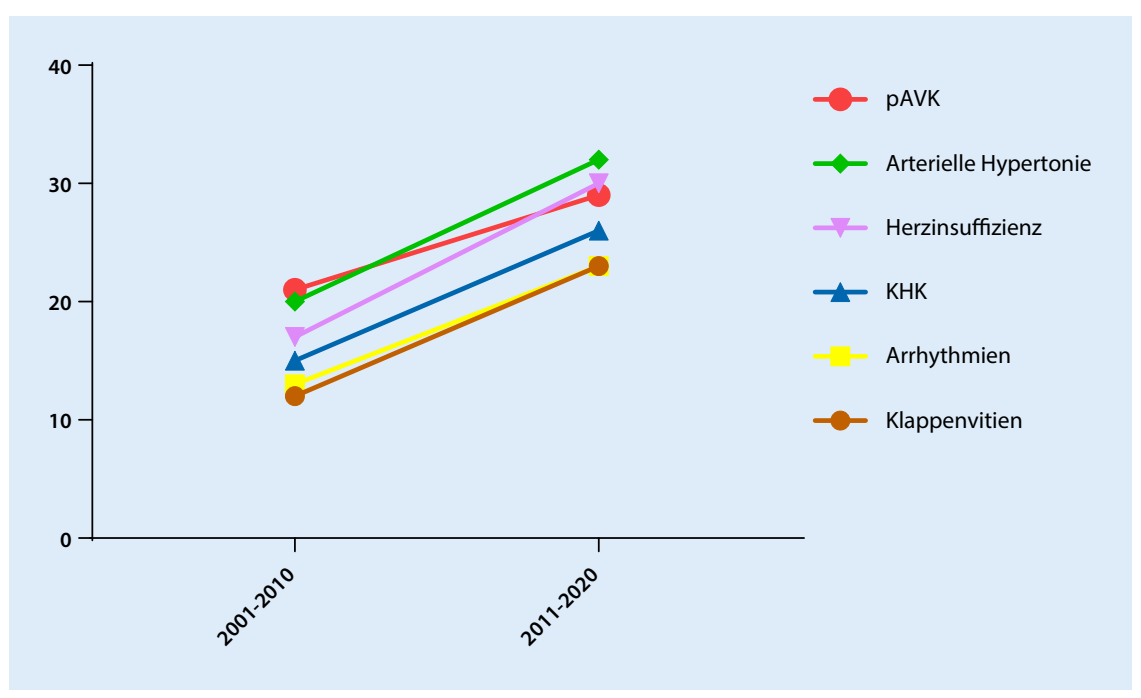

Abb. $5 \Delta$ Entwicklung der Geschlechterverteilung in der kardiologischen Forschung in Deutschland. Aufgeführt ist der Prozentsatz der weiblichen Autorinnen von 2001 bis 2010 sowie von 2011 bis 2020 getrennt für die verschiedenen Themenbereiche der Kardiologie. pAVK periphere arterielle Verschlusskrankheit, KHK koronare Herzkrankheit 


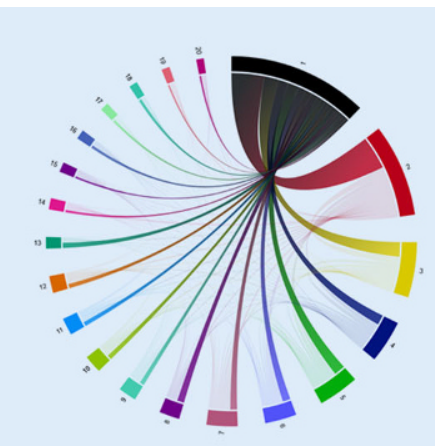

a

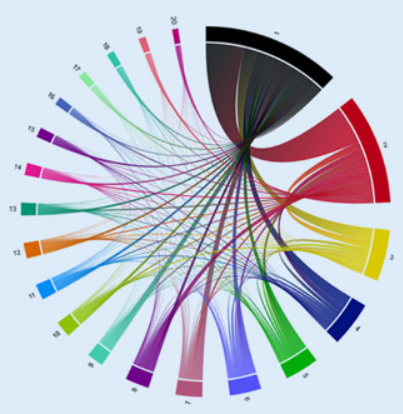

d

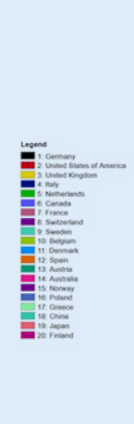

b

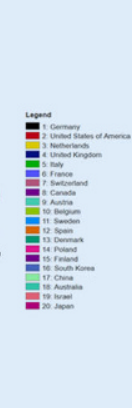

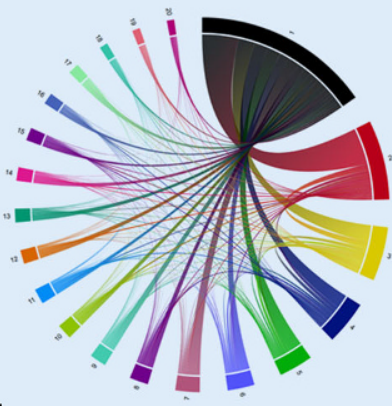

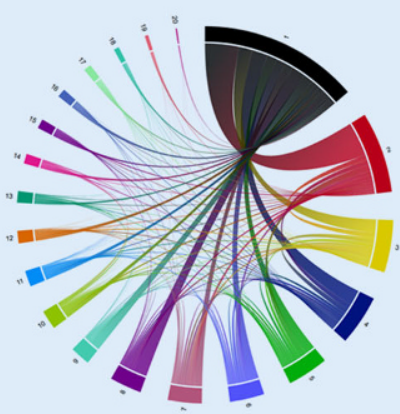

e

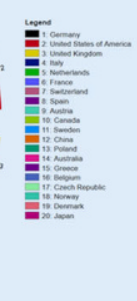

C

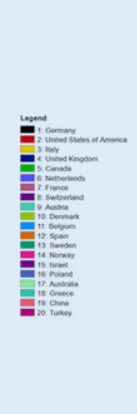

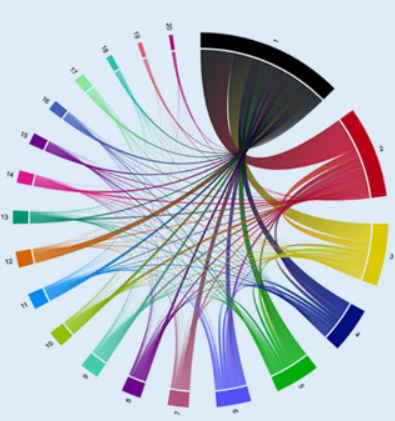
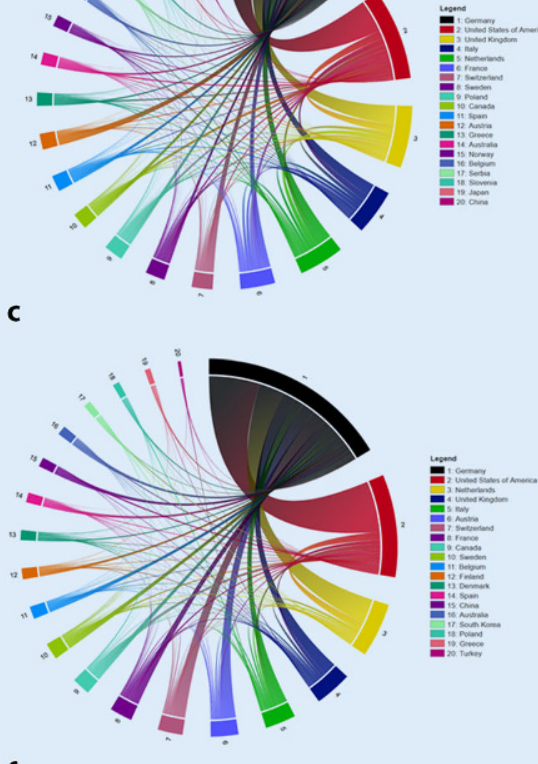

Abb. 6 \ Akkorddiagramm der internationalen Forschungskooperationen. Abgebildet sind die 20 häufigsten Kooperationspartner Deutschlands in Bezug auf alle Koautoren einer Publikation. Die Breite der Verbindungslinien ist proportional zum Ausmaß der Kooperation. a Arrhythmien. b Arterielle Hypertonie. c Herzinsuffizienz. d Koronare Herzkrankheit (KHK). e Klappenvitien. $\mathbf{f}$ Periphere arterielle Verschlusskrankheit (pAVK)

\section{Korrespondenzadresse}

Dr. Dominic Millenaar
Klinik für Innere Medizin III,
Kardiologie, Angiologie und
Internistische Intensivmedi-
zin, Universitätsklinikum des
Saarlandes und Medizinische
Fakultät der Universität des
Saarlandes
Kirrberger Str., Geb. 41.1,
66421 Homburg/Saar,
Deutschland
Dominic.Millenaar@uks.eu

Danksagung. Wir danken Alexander Flohr und Markus Dillmann für die Hilfe bei der Programmierung der Software SciPE.

\section{Einhaltung ethischer Richtlinien}

Interessenkonflikt. D. Millenaar, C. Ukena, A. Keller, F. Mahfoud und M. Böhm geben an, dass kein Interessenkonflikt besteht.

Für diesen Beitrag wurden von den Autoren keine Studien an Menschen oder Tieren durchgeführt. Für die aufgeführten Studien gelten die jeweils dort angegebenen ethischen Richtlinien.

\section{Literatur}

1. Statistisches Bundesamt (2020) Anzahl der Gestorbenen nach Kapiteln der ICD-10 und nach Geschlechtfür 2019

2. MillenaarD, Fehlmann T, Ukena C, ScholzS, Keller A, Mahfoud F, Böhm M (2020) Wissenschaftslandschaft Deutschland. Kardiologe 14:113-123

3. Millenaar D, Fehlmann T, Scholz SS, Pavlicek V, Flohr A, Dillmann M, Böhm M, Keller A, Mahfoud F, Ukena C (2020) Research in atrial fibrillation: a scientometric analysis using the novel web application sciPE. JACC Clin Electrophysiol 6(8):1008-1018. https://doi.org/10.1016/j.jacep. 2020.05.010

4. Grammes N, Millenaar D, Fehlmann T, Kern F, Böhm M, Mahfoud F, Keller A (2020) Research output and international cooperation among countries during the COVID-19 pandemic: scientometric analysis. J Med Internet Res 22:e24514

5. ScholzSS, Dillmann M, Flohr A, Backes C, Fehlmann T, Millenaar D, Ukena C, Böhm M, Keller A, Mahfoud F (2019) Contemporary scientometric analyses using a novel web application: the science performance evaluation (SciPE) approach. Clin Res Cardiol

6. Böhm M, Papoutsis K, Gottwik M, Ukena C (2015) Publication performance of women compared to men in German cardiology. int J Cardiol 181:267-269

7. Statistisches Bundesamt (2019) Bruttoinlandsproduktje Einwohnernach Bundesländern 